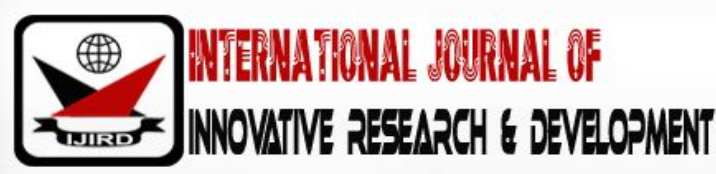

ISSN 2278 - 0211 (Online)

\section{Factors Affecting the Integrity of Oil and Gas Pipelines in Nigeria}

\author{
Bright, F. Ajibade \\ Chief Officer, Petroleum Training Institute, Nigeria \\ Ogunyemi E. Oluwole \\ Deputy Chief Officer, Petroleum Training Institute, Nigeria
}

\begin{abstract}
:
Basically, three major factors (EXD, AOC and COR respectively) have been identify as cause of pipeline integrity failure in the Nigeria pipeline networks. These three factors have various level of effects with distinct degree of influences. Under unrotated loading (when considering the data without introducing any effects), AOC and EXD serve as the most significant factors influencing pipeline integrity failure. AOC causes $94 \%$ of the pipeline integrity failure. EXD accounted for $92 \%$ of the cause of pipeline integrity failure. And COR accounted for $58 \%$ of the pipeline integrity failure. When rotational effect is introduced (i.e., considering the magnitude of the caused effect relationship of the factors), EXD takes the lead in all category. For instance, EXD accounted for the highest cause of integrity failure of the pipelines. EXD accounted for $92 \%$ and AOC, $78 \%$ for varimax rotation and EXD accounted for $100 \%$ and AOC, $65 \%$ for promax rotation. For quatim in rotation, EXD accounted for $101 \%$ and AOC, $58 \%$ with COR, $0 \%$. The results reveal that excavation damage (EXD) has the highest degree of contribution to the cause of pipeline integrity damage in the Nigeria pipeline networks. Similarly, all other causes (AOC) and corrosion (COR) also accounted for significant causes of pipeline integrity failure. We also found out that, both EXD and AOC are strongly (positive) correlated while COR has a weak positive correlation with both EXD and AOC.As a result of the aforementioned findings, it is therefore a known fact that, EXD (and AOC outside control (OCP)) in Nigeria are products of self-inflicted factor (leading to pipeline integrity failure) which has resulted in the current state of the communities and region of the Oil \& Gas producing wells. Consequently, Government need to work hand in hands (and compromise their stands when need be) with the affected community to put an end to the current state of EXD (and AOC_OCP) occurrences in order to ensure environmental and public safety of the region. Forceful implementation and military actions will increase the chances of EXD (and AOC_OCP) continuity. Social/ status improvement and community cooperation is the only way forward.
\end{abstract}

Keywords: Factors of pipeline integrity failure, confirmatory factor analysis, EXD, AOC and COR

\section{Introduction}

Pipeline operators are under severe financial and social pressure to avoid incidents that cause crude oil and natural gas leaks. The goal of pipeline producing company is to offer a pipeline service with zero pipeline failures by providing accurate and reliable pipeline inspection data that will help promote environmental and public safety (GE Oil \& Gas, 2017). Ensuring pipeline integrity is one major feature of a reliable pipeline company. However, in a situation where there are factors interfering or acting on the laid pipeline like pipeline linkages, corrosion etc., the integrity of such pipeline may be questionable. As a result, most pipeline companies have developed various technologies for detecting and monitoring the state of pipelines such as; to detect the external corrosion of oil and gas pipelines of buried pipelines. In this quest, they have been able to classify the factors which are based on the multi scale approach for onshore pipelines, taken into consideration the soil/ pipeline system as an electrochemical cell.

Usually, pipelines that are exposed to and interacting with various environmental elements are mostly exposure and prone to integrity damage. Apart from this, the chlorides and sulfates that are present in the soil can also cause the corrosivity of the soil and damage evolution of the pipeline/ soil system. Since resistivity has often been used as a major indicator of soil corrosivity, it is therefore ideal that the main methods for corrosion mitigation on underground pipelines are coatings and cathodic protection (Revie et al, 2015). Furthermore, other pipeline integrity services factors/ products that are often being considered by many pipeline manufacturers includes corrosion and metal loss characterization, crack management, geometry assessment solutions, integrity engineering, integrity planning and data management, line pig ability and outside force. 
Meanwhile, it is noteworthy to say that, corrosion is not the only factor militating against pipeline integrity. Corrosion can be view as a natural factor which is typically associated with the soil composition acting on the laid pipeline. Apart from natural factors, several other factors which are due to human error are mostly encounter and can mitigate the integrity of the pipeline. Factors such as excavation damage, incorrect operation, material failure etc., are company's/ human factors which are often the main determinants of pipeline quality and integrity operation.

In Nigeria, the integrity of pipelines laid or used across Nigeria (particularly Oil and Gas pipeline) pipeline network has been a point of discussion over time. The major issues are that, the pipeline quality and integrity are questionable. Many have argued that, beside the factors caused as a result of excavation damage, incorrect operation, material failure etc., there are other outside forces controlled by various situations which are not within the reach of the pipeline suppliers. And all these factors are threat to the pipeline integrity.

This paper examines the statistical comparison of these factors to know which one has the most influencing effect on pipelines integrity. The rest of this paper is structured as follows. Section 2 examines the relevant literatures and section 3 presents the data source and description. In section 4, we present the data analysis and presentation of the results. We conclude the paper in section 5.

\section{Literature Reviews}

With regulators scrutinizing pipeline projects due to the eroded reputation of the pipelines integrity, the reputation of the entire pipeline industry is at risk. This is why pipeline integrity has become the focus of discussion by many scholars. Several articles have look at various aspects related to pipeline integrity from a generic and also technological point of view, while at the same time discuss how these requirements for pipeline integrity management can be adequately addressed.

Lars (2010) affirms that, pipeline integrity is not just about preventing incidents, but is a holistic approach to the prevention, detection, and mitigation of commodity releases. He also noted that, increase in the exploration project demand around the world in order to uncover new hydrocarbon source has prompt the demand increases for more pipeline development. As a result of this, pipeline operators are under check and balances to avoid incidents that may result to release of commodity. By implication, there is need for safe practices and industrial strength prevention to ensure pipeline integrity practices.

Ramesh (2017), define pipeline integrity as the process that delivers necessary pipeline inspection methods, identification of hazard mechanisms, risk and consequence evaluations, and repair strategies. Covering relevant standards and processes for risk, assessment, and integrity management, this go-to reference provides the principles that guide these concepts enhanced with more critical regulatory information and easier organization between liquid and gas pipelines. Pipeline integrity has continued to provide engineers and plants managers a vital resource for keeping their pipelines and facilities safe and efficient. He proposed that setting an integrity management plan, updated with new information on corrosion control, gas \& liquid hydrocarbon and safe assessment program while properly characterizing impact of risk as well as understanding and apply all the latest and critical oil and gas pipeline standards, both U.S. and international-based is a core pipeline integrity value.

Su et al (2017), examine ten contemporary electric-resistance welded (ERW) pipe to investigate the influence of Charpy notch position in order to ensure the pipeline integrity in seam-weld region. They found out that the weld bond line (BL) region to be quite narrow and with microstructures similar to or finer than the adjacent regions and base steels. They also discover that, little texture was present in the BL regions after the ERW weld and post-weld heat treatment processes. Their result shows the existence of some variations in the through-thickness and the Charpy bond-line toughness show a relatively larger scatter. Their result has since been adopted in the Canadian pipeline standard as the basis for revising the ERW pipe toughness requirements to ensure continuous pipeline integrity. The major factor examine is improper design which could cause severe pipeline integrity issue.

Hryciuk et al (2017), examine girth weld integrity evaluation on a vintage gas pipeline. They discovered a leak on a girth weld transmission gas pipeline while recoating works were being performed. Failure analysis conducted showed that the leak was caused by a pre-existing defect in the girth weld which had not been evolving over time. They also observed that the link is caused as a result of particular stress condition to which the gas pipeline was subject during the recoating work, and the defect destabilized and causes progress failure. The main crux of their paper is that, they discover linkages/ cracks as a major factor impeding pipeline integrity.

Castiglia et al (2017), examines mitigation measures for the stability of pipelines in liquefiable soils. They proposed a new technique aimed at ensuring the stability of class (when the pipe is under the water table level, or floating due to earthquake forces) of structure crossing a liquefied area. They observed that one of the problems is the lack of standardization due to variable soil characteristics along the pipeline. They made an overview of the existing method, and new perspectives in mitigation measures are shown, in order to clarify the effectiveness of their application and provide some important tools for further developments. The major contribution of their work is that, they were able to proffer a modify approach to correct the stability of pipelines in liquefiable soil which may have been displaced by either a natural occurrence or human caused effect.

D’Alberto (2017), considers the engineering assessment for temporary wooden pipe supports to aid pipeline integrity. They observed that the destabilization forces on the skid supports are mainly due to the transverse deformation of the pipe string generated by the environmental thermal conditions. Consequently, if the stored energy exceeds the restraining 
capability of the skid supports, the pipeline, which is apparently in a normal position on the skids, may fall to the ground causing serious injuries or even fatalities. They propose that a pipeline temporarily supported by wooden cribbing blocks, showing how a thermal gradient between two sides of the pipe can lead to critical stability conditions of the pipe itself on the support so as to ensure commodity and pipeline integrity. They also conducted a sensitivity analysis to investigate the influence of pipe-string configuration, presence of bends, construction sequence, and daily sun irradiation. 3D FEM analyses were performed on the pipe and wooden skid assembly to understand failure mechanisms and to identify the most stable skid type in relation to their structural features and geometry. The crux of their work is basically to help pipeline and safety engineers to understand the limitations of wooden skid assembly so as to understand failure mechanism and to identify the most stable skid type in relation to their structural features and geometry. This will help forestall and correct material/ weld/ equipment failure.

Rushd \&Rahman (2017), examine the friction loss and hold-up ratio in the water-lubricated pipeline transportation of heavy oil. They adopted the core annular flow (CAF) method which is economically efficient and environmentally sustainable technology for transporting heavy oil and bitumen, especially compared with conventional methods such as truck hauling, dilution, and heating. Despite the major obstacle of CAF, they were able to validate and analyze with respect to the measured values of pressure losses in a CAF pipeline and introduced a new modeling approach that consider the underlying physics of CAF hydraulics. This help to understand the hydrodynamics that govern friction losses in CAF pipeline. This method will improve the pipeline integrity in respect to frictional losses associated with CAF pipelines.

Zhu (2016), examines a modified two-curve model for running fracture arrest design of high-strength transmission pipelines. He examines the traditional running-fracture control (RFC) and observed that the limitation which may downplay the pipeline integrity. The Battelle two-curve (BTC) model developed in the early 1970s has been widely used in the pipeline industry to determine arrest toughness in terms of the Charpy energy. A brief review of running-ductile-fracture control made shows that the Charpy- energy-based two-curve model remains viable and deserves further study. The BTC model is known to be only able to predict arrest toughness, but not arrest distance. In order to fulfil technical gap, they developed a modified twocurve (MTC) model and a fracture-arrest- distance model in reference to the Charpy energy. The MTC model coupling with the arrest-distance algorithm can predict both arrest toughness and arrest distance in one simulation for a single pipe or a set of multiple pipes with given toughness. With this new modified approach, pipeline integrity can be achieved. Yasser \& Bahai (2016), examine decision-support tools for selection of pipeline corrosion coatings. They suggest that a suitable coating is one with high performance and durability; low surveillance and maintenance needs; easy application with no health implications; and finally, is cost effective. They observe that, it won't be difficult to imagine that one type of coating would not be suitable for all situations. In their paper, they present three methods for a pipeline corrosion- coating selection process, where judgment of more than one expert can be aggregated to reach consensus. Firstly, they consider a few multi-criteria decision-making methods, and then compare three different decision-support tools which are; simple addition of weights, Pugh's Matrix, and Borda's algorithm, where the judgment of more than one expert can be elicited and combined. Criteria for selection of the coating are then identified, and a panel of experts are asked to rate a number of candidate coatings against these criteria. They combined their voting using Borda's algorithm and Pugh's matrix. The contribution of their paper is proffering the best coating method to salvage corrosion of pipelines and thus ensuring pipeline integrity.

Based on literatures, seven (7) major factors have been identified. These factors are; corrosion (COR), excavation damage (EXD), incorrect operation (INOP), material/ weld/ equipment failure (MATFAL), natural force damage (NFD), other outside force damage (OUTFD) and all other causes (AOC). The crux of this paper is to examine which of these factors has the most influencing effect on pipelines integrity in Nigeria scene.

\section{Data Description}

We extracted data on the seven major factors mentioned above from the Nigeria Oil \& Gas data reservoir. The data cover pipeline integrity data from 1996 to 2016. Each of the factors were observed base on the number of reported occurrences on the pipeline network across the period of study.

\section{Data Analysis and Presentation of Results}

In order to achieve the main objective of this paper, we consider a confirmatory factor analysis procedure to identify the factor among these factors that has the most influencing effect on the pipeline integrity based on Nigeria data. First, we consider the descriptive study of the data collected and we concluded this section with the interpretation of the result findings.

\subsection{Descriptive Study}

We observe that, the most influencing factor of the pipeline integrity is the excavation damage (EXD) followed by all other causes (AOC). Averagely, there are 10 cases of AOC, 2 - 3 cases of COR, 13 - 14 cases of EXD, 5 cases of INOP, 3 - 4 cases of MATFAL, 2 cases of NFD and 5 - 6 cases of OUTFD. 
Table 1 gives the detailed descriptive result of the data.

\begin{tabular}{|c|c|c|c|c|c|c|c|}
\hline Statistics & AOC & COR & EXD & INOP & MATFAL & NFD & OUTFD \\
\hline Min. & 2.00 & 0.00 & 5.00 & 1.00 & 1.00 & 0.00 & 0.00 \\
\hline 1st Quart. & 6.00 & 1.75 & 7.00 & 3.00 & 2.00 & 1.00 & 1.75 \\
\hline Median & 8.50 & 2.50 & 9.50 & 5.00 & 3.00 & 2.00 & 6.50 \\
\hline Mean & 10.05 & 2.30 & 13.25 & 5.05 & 3.35 & 2.05 & 5.55 \\
\hline 3rd Quart. & 13.50 & 3.00 & 17.50 & 6.25 & 4.25 & 3.00 & 8.25 \\
\hline Max & 23.00 & 6.00 & 31.00 & 11.00 & 7.00 & 6.00 & 14.00 \\
\hline
\end{tabular}

Table 1: Summary Statistics of the Data

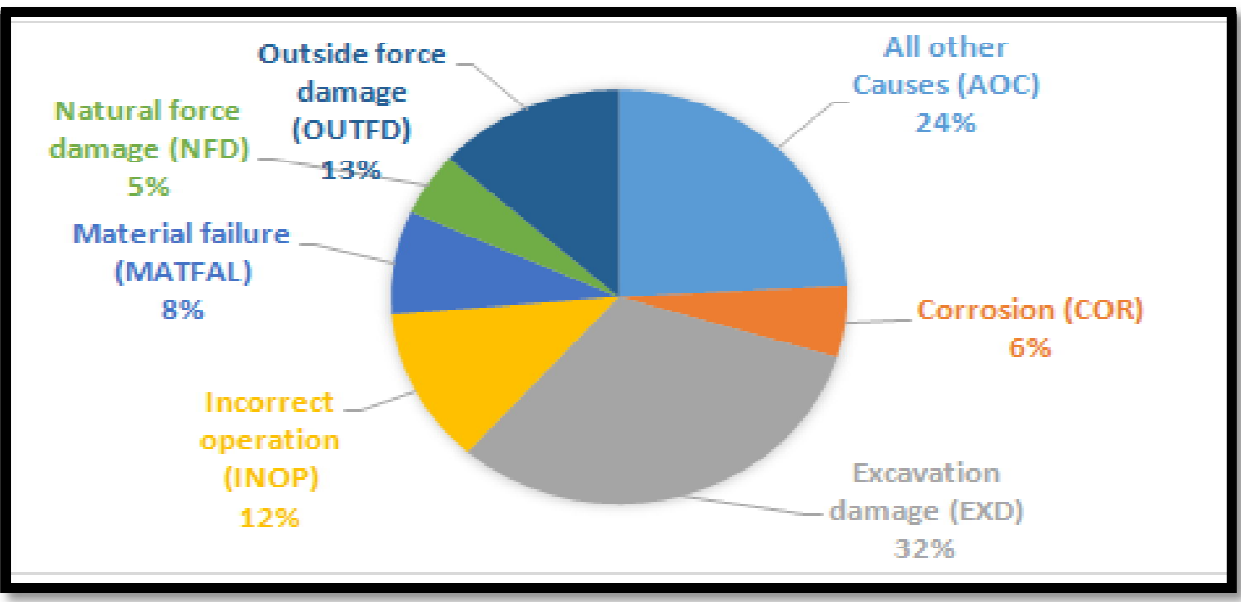

Figure 1: Factor of Pipeline Integrity: Nigeria Oil \& Gas

Pipeline between 1997 and 2016

Further percentage summary of the data reveal that, EXD is the most reported cases of pipeline integrity failure. Figure 1 presents the pictorial detail of the summary.

We also consider the distribution of the data across each of the factor using box and whisker plot for the distribution comparison. The result reveals that, both the EXD and AOC distinctly skewed positively. This implies that, there are higher occurrences (increase yearly) of these two factors influencing the pipeline integrity failure. OUTFD is negatively skewed, which implies that, it reduces across the year. All other factors featured show a closely symmetry feature with an evidence of outlier in COR. Refer to Figure 2 for detailed result.

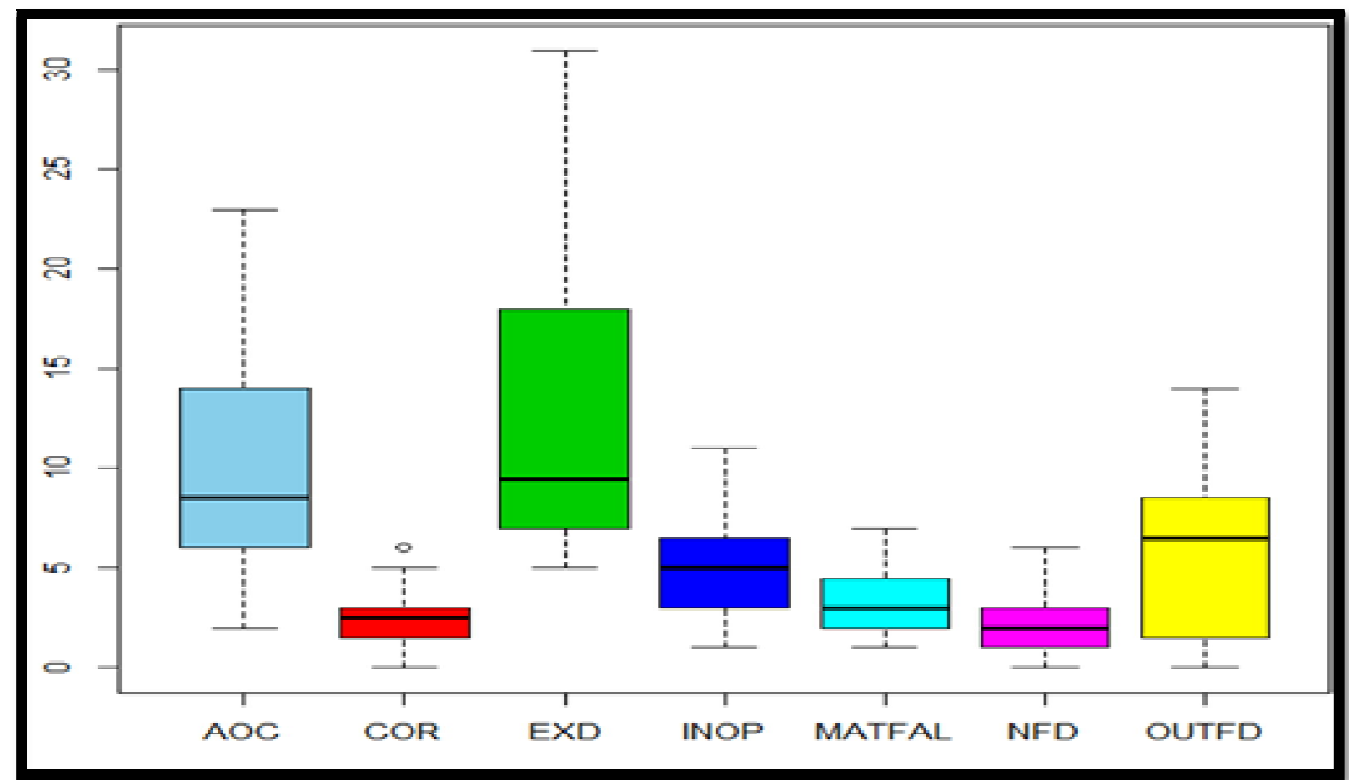

Figure 2: Distribution of the Factor Occurrences between 1996 And 2016 


\subsection{Factor Correlation Matrix}

We first consider the correlation statistics of these factors and see how closely related they are to one another. EXD and $\mathrm{AOC}$ have a very strong positive correlation. By implication, the occurrence of any of these two factors tends to prompt the occurrence of the other. There is a high chance that, they are product of the same process. Besides these two factors with strong positive relationship, the relationships between other factors (between AOC and INOP, MATFAL, NFD) are very weak positive relationship.

COR also show a mild strong (0.5737) positive relationship with AOC and weak (0.3776) positive relationship with EXD. Similarly, MATFAL has a weak positive relationship with COR and INOP respectively. Table 2 presents detailed result of the factors correlation matrix.

\begin{tabular}{|c|c|c|c|c|c|c|c|}
\hline & AOC & COR & EXD & INOP & MATFAL & NFD & OUTFD \\
\hline AOC & 1 & 0.57372 & 0.823902 & 0.381007 & 0.215764 & 0.176188 & -0.6904 \\
\hline COR & & 1 & 0.377675 & 0.27962 & 0.227933 & -0.00573 & -0.63586 \\
\hline EXD & & & 1 & 0.28392 & -0.01048 & -0.08829 & -0.62622 \\
\hline INOP & & & & 1 & 0.257557 & 0.141662 & -0.15498 \\
\hline MATFAL & & & & & 1 & 0.011454 & -0.0534 \\
\hline NFD & & & & & & 1 & 0.088951 \\
\hline OUTFD & & & & & & & 1 \\
\hline
\end{tabular}

Table 2: Factors Correlation Matrix

\subsection{Confirmatory Factor Analysis}

The factor loading retain only three of its components. These three factor components are significant at $5 \%$ level of significance. The scree plot (Figure 3) is the pictorial illustration of the retained factor components and Table 3 presents the result.

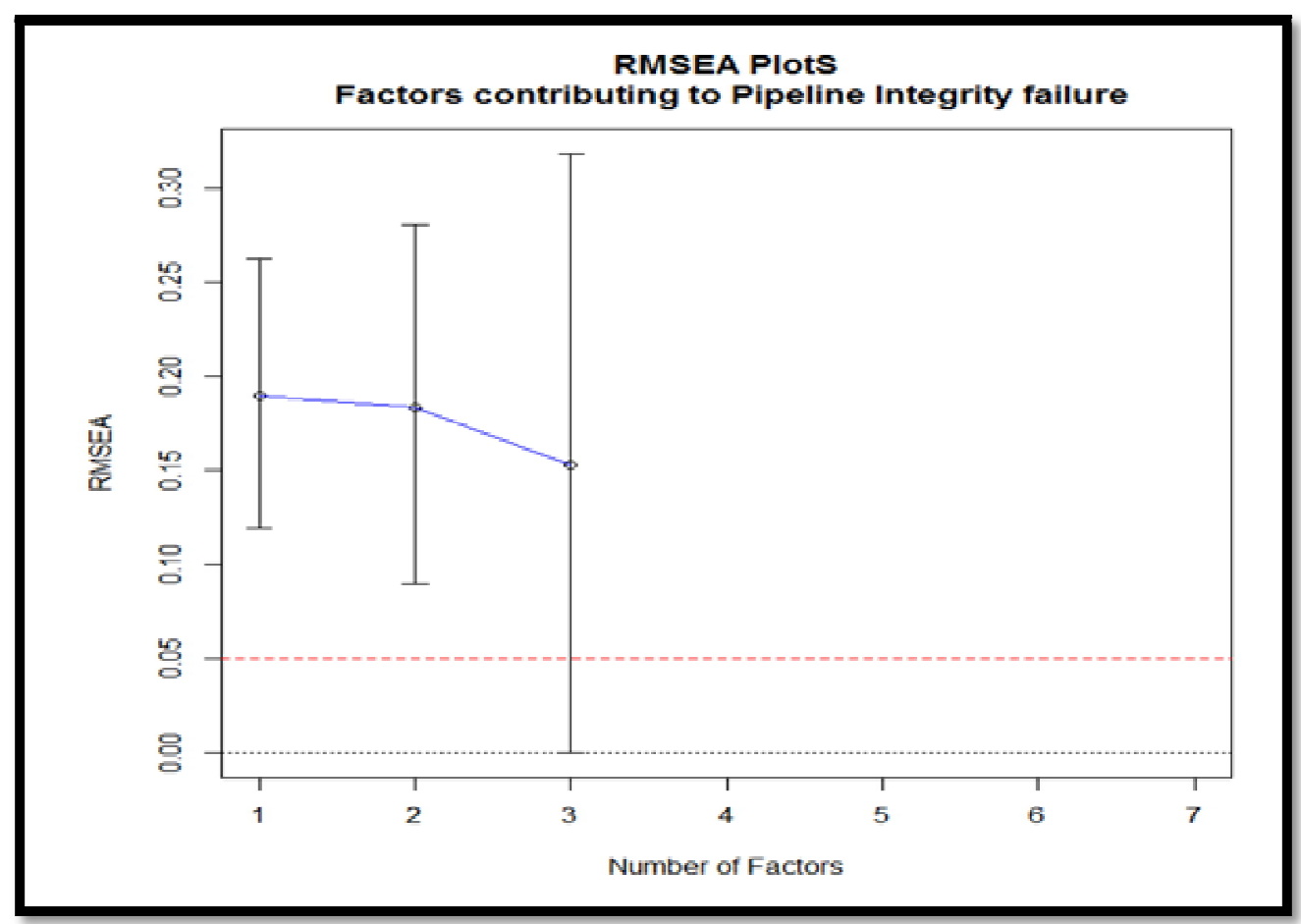

Figure 3: Scree Plot of the Factor Components

The first factor component retain has the highest loading compare to the other two factor components. As a result, factor component 1 has more influence in determining which of the factors has the most influence on the pipeline integrity failure. Although, the three factor components will significantly explain the factors influence on the integrity failure of the pipelines. 


\begin{tabular}{|c|c|c|c|c|c|c|c|}
\hline Factors & Cum. Eigen & Chi-Square & DF & P. Value & RMSEA.Pt & RMSEA. Lo & RMSEA.Hi \\
\hline 1 & 3.073241619 & 38.6238696 & 14 & 0.000417 & 0.189459 & 0.119624 & 0.261957 \\
\hline 2 & 4.312499636 & 21.14159604 & 8 & 0.006781 & 0.183097 & 0.089839 & 0.280113 \\
\hline 3 & 5.321911278 & 6.424606095 & 3 & 0.092684 & 0.152632 & 0 & 0.318021 \\
\hline 4 & 6.067614097 & - & -1 & - & - & - & - \\
\hline 5 & 6.652146498 & - & -4 & - & - & - & - \\
\hline 6 & 6.913427892 & - & -6 & - & - & - & - \\
\hline 7 & 7 & - & -7 & - & - & - & - \\
\hline
\end{tabular}

Table 3: Factor Component Retain Information

In order to ascertain or to establish the effect/ influence of the factor(s) on the integrity failure of the pipeline, we exhausted all available procedure to identify which of the factors that has the most significant influence in relation to the cause of the pipeline integrity failure. Emphasis is laid on the factor component 1 because; it has the most significant influence and has the highest eigen value compare to other factor components loaded. This significant influence (of the first factor component) is shown in the RMSEA plot (Fig. 4).

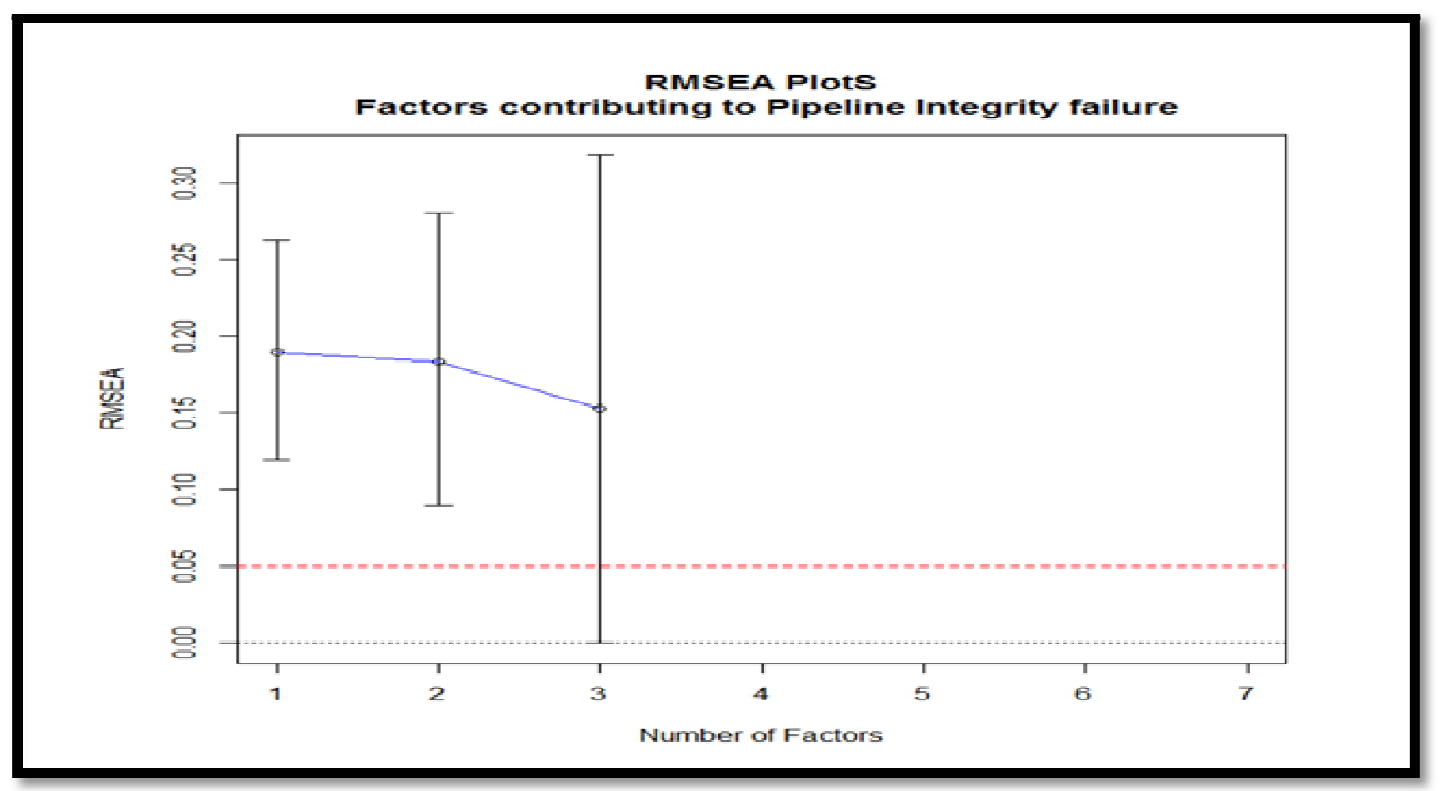

Figure 4: RMEA Plot of the Factor Components Loaded

We consider six (6) available rotation loading methods to identify which of the factors has the highest influence on the cause of integrity failure of the pipeline network. We present the result in Table 4. Under unrotated loading (when considering the data without introducing any effects), AOC and EXD serve as the most significant factors influencing pipeline integrity failure. AOC causes $94 \%$ of the pipeline integrity failure. EXD accounted for $92 \%$ of the cause of pipeline integrity failure. And COR accounted for $58 \%$ of the pipeline integrity failure.

When rotational effect is introduced (i.e., considering the magnitude of the caused effect relationship of the factors), EXD takes the lead in all category. For instance, EXD accounted for the highest cause of integrity failure of the pipelines. EXD accounted for $92 \%$ and AOC, 78\% for varimax rotation and EXD accounted for $100 \%$ and AOC, $65 \%$ for promax rotation. For quatimin rotation, EXD accounted for $101 \%$ and AOC, 58\% with COR, $0 \%$.

\begin{tabular}{|c|c|c|c|c|c|c|c|c|c|c|}
\hline \multicolumn{10}{|c|}{ Factors of Pipeline Integrity failure } \\
\hline Rotation Loadings & AOC & COR & EXD & OUTFD & INOP & MATFAL & NFD & SS loading & Prop. Var & Cum. Var \\
\hline Unrotated & & & & & & & & & & \\
\hline Factor 1 & 0.94 & 0.58 & 0.92 & -0.83 & 0.31 & & & 2.87 & 0.41 & 0.41 \\
\hline Factor 2 & 0.31 & - & & & & 0.40 & 0.50 & 0.67 & 0.10 & 0.51 \\
\hline Factor 3 & - & -0.32 & & 0.54 & & & & 0.54 & 0.08 & 0.58 \\
\hline Varimax & & & & & & & & & & \\
\hline Factor 1 & 0.78 & & 0.92 & -0.34 & 0.33 & & & 1.71 & 0.24 & 0.24 \\
\hline
\end{tabular}




\begin{tabular}{|c|c|c|c|c|c|c|c|c|c|c|}
\hline \multicolumn{9}{|c|}{ Factors of Pipeline Integrity failure } \\
\hline Factor 2 & 0.52 & 0.63 & 0.34 & -0.94 & & & & 1.68 & 0.24 & 0.48 \\
\hline Factor 3 & 0.34 & & & & & 0.44 & 0.51 & 0.69 & 0.10 & 0.58 \\
\hline Promax & & & & & & & & & & \\
\hline Factor 1 & 0.65 & & 1.00 & & & & & 1.53 & 0.22 & 0.22 \\
\hline Factor 2 & & -0.61 & & 0.99 & & & & 1.44 & 0.21 & 0.42 \\
\hline Factor 3 & 0.38 & & & & & 0.42 & 0.54 & 0.78 & 0.11 & 0.54 \\
\hline Quatimin & & & & & & & & & & \\
\hline Factor 1 & 0.58 & & 1.01 & & & & & 1.47 & 0.21 & 0.21 \\
\hline Factor 2 & & -0.62 & & 1.00 & 0.30 & & & 1.46 & 0.21 & 0.42 \\
\hline Factor 3 & 0.48 & & & & & 0.42 & 0.54 & 0.86 & 0.12 & 0.54 \\
\hline Orthogonal Bifactor & & & & & & & & & & \\
\hline Factor 1 & 0.88 & 0.55 & 0.93 & -0.86 & & & & 2.76 & 0.39 & 0.39 \\
\hline Factor 2 & 0.47 & & & & & & 0.51 & 0.78 & 0.11 & 0.51 \\
\hline Factor 3 & & -0.36 & 0.35 & 0.51 & & 0.40 & & 0.53 & 0.08 & 0.58 \\
\hline Oblique Bifactor & & & & & & & & & & \\
\hline Factor 1 & 0.78 & 0.55 & & -0.47 & 0.34 & 0.38 & 0.40 & 1.63 & 0.23 & 0.23 \\
\hline Factor 2 & 0.61 & & 0.95 & & & & & 1.37 & 0.20 & 0.43 \\
\hline Factor 3 & & -0.45 & & 0.88 & & & & 1.09 & 0.16 & 0.59 \\
\hline
\end{tabular}

Table 4: Factors Influence Summary

AOC: All Other Causes, COR: Corrosion, EXD: Excavation Damage, INOP: Incorrect Operation,

MATFAL: Material Failure, NFD: Natural Force Damage, OUTFD: Other Outside Force Damage

\section{Summary \& Conclusion}

\subsection{Summary}

Basically, three major factors (EXD, AOC and COR respectively) have been identify as cause of pipeline integrity failure in the Nigeria pipeline networks. These three factors have various levels of effects with distinct degree of influences. The result reveals that excavation damage (EXD) has the highest degree of contribution to the cause of pipeline integrity damage in the Nigeria pipeline networks. Similarly, all other causes (AOC) and corrosion (COR) also accounted for a significant causes of pipeline integrity failure.

Having said this, EXD is the most pronounced factor which is associated as the cause of pipeline integrity failure. Just like it is observed in other nations (for instance, US), EXD is observe as the major threat of pipeline integrity."Through about 2010, excavation damage was the leading cause of all significant pipeline incidents, resulting in many deaths and injuries, as well as substantial property damage. While excavation damage is no longer the leading cause of significant pipeline incidents, it is still a major cause, and is still the second leading cause of serious incidents (those that cause death or injury requiring hospitalization)" - (US, Pipeline safety Trust, 2015.)

Meanwhile, the cause player of EXD varies across board. In the US and some other parts of the world, EXD is seen as accidental damage to the pipe or its coating that is caused by someone inadvertently digging into a buried pipeline. Thus, EXD is mostly unintentional and is usually resulted from digging, grading, trenching, boring activities or road constructions (in rare cases). In Nigeria, EXD are mostly intentional. Evidently, pipeline vandalism is a big challenge to the Nigeria government and threats from the Niger-Delta Avengers is one of the numerous obvious account of this. As a result of this, EXD is the major threatening factor of pipeline integrity in Nigeria since it occurrence cannot (at the moment) be predicted. Unlike in other nations (where EXD rate has decreased/ descended over time), the EXD rate in Nigeria increases across the year and it is mostly influenced by several local forces or factors.

The all other cause (AOC) perhaps are other forces which are within and outside the control of the pipeline producers. The AOC outside control (AOC_OCP) may be a product of natural disasters (such as, earthquake, tsunami etc.), war or acts of terrorism. In Nigeria, both EXD and AOC are positively correlated (Table 1). For instance, series of pipeline vandalism (EXD) are products of acts of terrorism (AOC) such as; groups revolting (fighting) against government by destroying Oil \& Gas pipelines. This has resulted in huge commodity waste and causes Oil spills in the region. By implication, these two factors have the most significant influence on the pipeline integrity of the Nigeria pipeline networks. The AOC within control (AOC WCP) may result from design failure from the pipeline producers. And such may cause pipeline linkages and other commodity waste.

\subsection{Conclusion}

It is therefore a known fact that, EXD (and AOC_OCP) in Nigeria are products of self-inflicted factor (leading to pipeline integrity failure) which has resulted in the current state of the communities and region of the Oil \& Gas producing wells. As a 
result of this, Government need to work hand in hands (and compromise their stands when need be) with the affected community to put an end to the current state of EXD (and AOC OCP) occurrences in order to ensure environmental and public safety of the region. Forceful implementation and military actions will increase the chances of EXD (and AOC_OCP) continuity. Social/ status improvement and community cooperation is the only way forward.

Unlike EXD and AOC, COR is a problem mainly associated with the pipeline operators/ producers. All categories of COR that causes pipeline integrity may be attributed to the type coating used or adopted by the pipeline producers coupled with level of compliance with international coating standard. Consequently, pipeline operators should endeavour and ensure that, they implement all international standard (of coating and safety) on pipelines used in the Nigeria Oil \& Gas industry and all wear out pipelines are replaced and proper pipeline inspection is done time to time. More so, all inspection data should be well analyzed in order to promote environmental and public safety.

\section{References}

i. D’Alberto Diego, Dr Agostino Napolitano, Salvatore Morgante, and Angelo Rosato (2017), Engineering assessment for temporary wooden pipe supports, available at: http:/ / www.j-pipe-eng.com/ abstract.cfm?cat_no=2413s, accessed 14th Dec., 2017

ii. GE Oil \& Gas (2017), Pipeline Integrity Service., available at: https:/ / www.geoilandgas.com/ pipelinestorage/ pipeline-integrity-services, accessed 14th Dec., 2017.

iii. Lars Larsson (2010), Pipeline Integrity: Best Practices to prevent, Detect, and Mitigate Commodity Releases., available at: http:/ / www.ogj.com/ whitepapers/ 2015/ 04/ pipeline-integrity-best-practices-to-prevent-detect-and-mitigatecommodity-releases.whitepaperpdf.render.pdf, accessed 14th Dec., 2017.

iv. Massimina Castiglia, Salvatore Morgante, Dr Agostino Napolitano, and Prof. Filippo Santucci de Magistris (2017), Mitigation measures for the stability of pipelines in liquefiable soils, available at: http:/ / www.j-pipeeng.com/ abstract.cfm?cat no=2409s, accessed 14th Dec., 2017.

v. Pedro M. Hryciuk, Jose A. Minellono, and Verónica Domínguez (2017), Girth weld integrity evaluation on a vintage gas pipeline, available at: http:/ / www.j-pipe-eng.com/ abstract.cfm?cat no=2410s, accessed 14th Dec., 2017.

vi. Ramesh Singh (2017), Pipeline integrity, management and risk evaluation 2nd edition, available at: https:/ / www.elsevier.com/ books/ pipeline-integrity/ singh/ 978-0-12-813045-2, accessed 14th Dec., 2017

vii. Sayeed Rushd and Aziz Rahman (2017), A study on friction loss and hold-up ratio in the water-lubricated pipeline transportation of heavy oil, available at: http:/ / www.j-pipe-eng.com/ abstract.cfm?cat_no=2412s, accessed 14th Dec., 2017.

viii. Sirous Yasser and Hamid Bahai (2016), Decision-support tools for selection of pipeline corrosion coatings, available at: http:/ / www.j-pipe-eng.com/ Abstract.cfm?cat no=2406s, accessed 14th Dec., 2017.

ix. Su Xu, Andrew Laver, Jim Gianetto, Jie Liang, William R. Tyson, and Shinya (Matt) Matsuno (2017), Charpy toughness of ERW seam welds, available at: http:/ / www.j-pipe-eng.com/ abstract.cfm?cat_no=2411s, accessed 14th Dec., 2017.

x. US, Pipeline safety Trust (2015), Excavation Damage Prevention, available at: http:/ / pstrust.org/ wpcontent/ uploads/ 2015/ 09/ 2015-PST-Briefing-Paper-07-Excavation-Damage-Prevention.pdf, accessed 15th Dec., 2017.

xi. Winston R. Revie, Homero Castaneda and Omar Rosas (2015), Oil and Gas: Integrity and Safe Handbook. Chapter 2, external corrosion of pipeline in soil. Available at: http:/ / onlinelibrary.wiley.com/ doi/ 10.1002/ 9781119019213.ch20/ summary, accessed 14th Dec., 2017.

xii. Xian-Kui Zhu (2016), A modified two-curve model for running fracture arrest design of high-strength transmission pipelines. Journal of Pipeline Engineering, vol. 16, available at: http:/ / www.j-pipeeng.com/Abstract.cfm?cat_no=2390s, accessed 14th Dec., 2017. 